\title{
A macrobending singlemode fiber refractive index sensor for low refractive index liquids
}

\author{
Pengfei Wang,* Yuliya Semenova, Yong Li, Qiang Wu, Gerald Farrell \\ Photonics Research Center, School of Electronic and Communications Engineering, Dublin Institute of Technology, \\ Kevin Street, Dublin 8, Ireland
}

Received March 25, 2010; revised April 29, 2010; accepted June 1, 2010; published June 30, 2010

\begin{abstract}
An all-fiber refractive index sensor with a simple configuration is proposed and investigated. The proposed fiber refractometer consists of a full-loop structure of bare high-bend loss singlemode fiber-1060XP, employing the well-known whispering gallery mode (WGM) effect induced by the evanescent wave excitation to measure the refractive index of an external medium. A competitive sensitivity is achieved (average $725.76 \mathrm{~nm} / \mathrm{RIU}$ at a bend diameter of $19.3 \mathrm{~mm}$ ) compared with other fiber-optic sensors, plus benefits resulting from simplicity, low cost and ease of fabrication.
\end{abstract}

In recent years, there has been a growing interest in the study of refractive index (RI) sensors, which are aimed at detection, diagnosis, and determination in the fields of liquid food and water-quality control, health, safety, and environmental monitoring. Fiber sensors have attracted tremendous interest for a range of sensing applications due to their well-known advantages such as immunity to electromagnetic interference, small size, light-weight, high temperature resistance, corrosion resistance, high sensitivity and electrically passive operation. A number of fiber RI sensors have been developed such as long period fiber gratings (LPFG) [1], fiber Bragg gratings (FBG) [2], fiber surface plasmon resonance (SPR) devices [3] and fiber-based Fabry-Perot interferometers [4] etc. Fabrication of such fiber refractometers can be costly increasing the cost of the entire sensing system. Therefore, there is a need for an optical fiber RI sensor with a simple configuration, which is easy to fabricate and demonstrates a high efficiency and can be used as a successful RI sensor. An RI sensor based on a macrobend fiber is a promising candidate given the ease with which the sensor head can be fabricated.

We have shown previously that fiber macrobending loss can be utilized for RI sensing applications [5] to measure the RI of petrochemical liquids which have a RI range of 1.46 1.56, greater than that of the fiber cladding. For such an RI range Whispering Gallery Mode (WGM) effects [6] do not occur at the fiber bend and the bend loss versus RI behavior is predictable.

However, for biosensing applications, the various organic solvents employed as solutions or interaction

*E-mail: pengfei.wang@dit.ie media have RI values that lie in the range 1.33 1.40, for example, deionized water (1.333@589.3nm) and acetone (1.359@589.3nm). Such RI values are below the RI of fused silica (1.458@589.3nm), which is the primary material making up the fiber cladding and core, resulting in WGM effects. In this paper, we propose a fiber RI sensor for low RI liquids that operates on the principle of exciting the WGMs formed in the bent bare singlemode fiber, where the peak shift of wavelength at which the WGMs excitation occurs serves as a measure of the RI of the external medium. This involves a significantly different operating principle and measurement approach, by comparison to fiber RI sensors where the RI values under test are greater than those of the cladding.

We numerically analyze the proposed RI sensor with a full-vectorial beam propagation method and experimentally evaluate the performance of the sensor. Our results show that the sensor can provide a resonance peak shift suitable for a high precision RI measurement and a competitive sensitivity comparable to that of existing FBG or SPR sensors. The proposed macrobending fiber based refractometer provides a competitive sensitivity (average $725.76 \mathrm{~nm} / \mathrm{RIU}$ at a bend diameter of $19.3 \mathrm{~mm}$ ). By comparison with existing fiber based RI sensors, the proposed sensor allows for measurement of low RI value liquids, while offering the advantages of other macrobend fiber based sensors, such as ease of fabrication for the refractometer head. Preliminary experimental results show the possibility of distinguishing between different organic solvents used in biosensing on the basis of RI value.

In a previously published investigation of WGM effects in a bent fiber [7], a bare bent singlemode fiber-SMF28 was employed for temperature sensing. Because SMF28 is a low bend loss fiber, in order to obtain a deep WGM resonance peak, the fiber needs to be bent at a small bend radius; however, a small bend radius increases the risk of breakage. To reduce the bending induced internal stress and to ensure the mechanical stability for the bent fiber, partial etching of the fiber cladding has been demonstrated as an effective solution, but this increases the fabrication difficulty and cost. In this paper, a high bend loss fiber-1060XP is employed; this avoids the need for partial 
etching of the fiber and leads to sufficiently deep WGM resonance peaks resulting from bending at relatively large bending diameters.

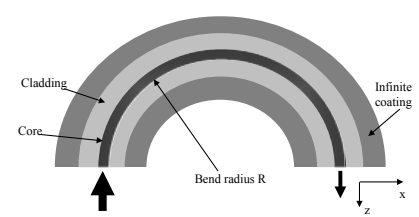

(a)

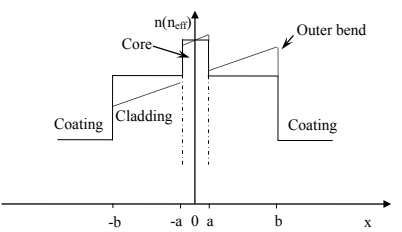

(b)
Fig.1. a) Structure of a bending fiber consisting of core, cladding and infinite coating; b) RI profile of the straight fiber (solid line) and effective RI profile with a conformal mapping of the bending fiber (dashed line).

Figure 1(a) presents a schematic structure of a bending fiber consisting of a core, cladding and a coating. Figure 1 (b) shows RI profile of the straight fiber (solid line) and effective RI profile with a conformal mapping of the bending fiber (dashed line), from which one can see that the RI of the coating is significantly lower than the refractive indices of the fiber core and cladding. Here we consider the coating as an infinitely thick layer with a RI equal to that of the surrounding medium. Since the "coating" in Fig. 1 (b) is a liquid, bending does not change the RI distribution of this infinite coating. For this structure, the strong reflection of the radiated field occurring at the interface between the cladding and the infinite coating has a significant effect on the bend loss performance and forms a very strong interference spectral response over a large wavelength range due to the WGM effects caused by reflections from the glass/liquid interface. The combined cladding and core of the bent fiber can be regarded as a few-mode fiber or a long-period grating.

To examine the modal interference process in the cladding region of a bent fiber, we use the implicit finite difference beam propagation method (FDBPM), as it allows a 3-dimesional (3-D) full vectorial formulation in the paraxial approximation [8]. As an example, a 2-dimensional (2-D) quasi mode field distribution for the case of bare bending 1060XP in air is presented in Fig. 2. It can be seen that the reflected field by the interface of cladding layer and air (infinite coating) is strongly coupled with the guided mode within the fiber core region along the direction of light propagation. From the figure, one can also see that the single quasi-guided mode cannot be observed clearly in this case due to interference from the cladding mode and that the intensity of the cladding mode is significantly larger than that of the core mode.

In this paper, the proposed fiber refractometer sensor consists of a single loop structure of bare macrobending fiber with a defined bending radius utilizing WGM resonance effects in the bare fiber section. As an example, we measured using a broadband source and an optical spectrum analyzer the bend loss of the bare 1060XP fiber with a diameter of $19 \mathrm{~mm}$ (the bend length is one turn) over a wavelength range from 1500 to $1600 \mathrm{~nm}$. The bare 1060XP fiber was bent in free space to form a small $360^{\circ}$ bend, in a "knot-like" fashion for mechanical stability. The corresponding experimental results are presented in Fig. 3. For the case of fiber core-cladding-air, the reflection of the radiated field at the interface between the cladding layer and air has a significant impact on the bend losses.

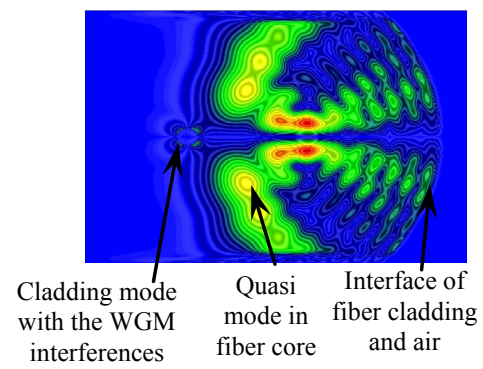

Fig. 2. 2-D quasi mode pattern for curvature diameter of $19 \mathrm{~mm}$ for the bare bending 1060XP in air, simulated by a 3-D FDBPM method [9].

From Fig. 3, one can see that significant WGM resonances occur over the investigated wavelength range. The spectral transmission response in Fig. 3 shows two major loss peaks over the measured wavelength range. If the bare bending fiber section is immersed in a liquid after the coating is removed, the wavelength positions of the WGM resonance peaks become a function of the liquid RI. To maximize RI measurement accuracy the deepest WGM resonance is chosen, in this case the first loss peak so that by measuring the shift in the peak wavelength, a change in the RI of the surrounding liquid can be determined. It is found that for curvature diameters above $20 \mathrm{~mm}$ the intensity of WGMs was too low to provide a useful sensitivity; while for the diameters below $18 \mathrm{~mm}$ the risk of fiber breakage due to excessive stress was too high.

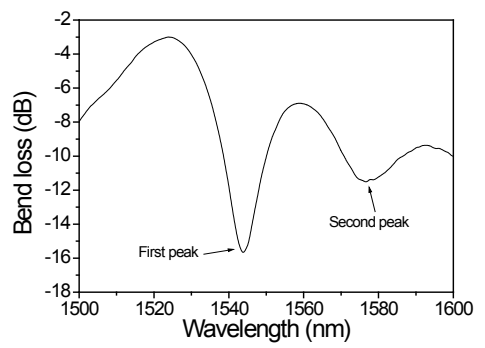

Fig. 3. Measured bend loss of bare 1060XP fiber in air at the bend diameter of $19 \mathrm{~mm}$.

For experimental studies of the proposed refractometer the fiber bend diameter used was $19 \mathrm{~mm}$ and the experimental setup is shown in Fig. 4. The polymer coating layers of 1060XP fiber were stripped by a mechanical stripper. The fiber sensor head was formed from a single $360^{\circ}$ loop tied as a knot in free space. The fiber knot is glued to form a stable macrobending fiber refractometer head. 


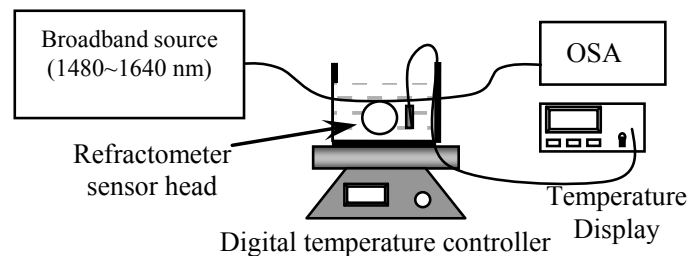

Fig. 4. Experimental setup for investigation of the proposed fiber refractometer.

To evaluate the performance of the proposed fiber refractometer for RI measurement, the fiber sensing device was tested using a range of organic solvents including methanol, acetone, acetic acid, ethanol, butyl formate, butanol and deionized water at room temperature. The RIs of the organic solvents employed were also optically measured for comparison using an Abbe refractometer with a resolution of $10^{-3}$ RI units (RIU). The corresponding transmission spectra are shown in Fig. 5(a). From the figure, the wavelength position of the first resonance peak shifts towards longer wavelengths as a result of the increase of RI, of the various organic solvents. Thus, the RI difference of various solvents can be accurately measured relative to some reference RI, such as de-ionized water. It is observed that the resonance loss peaks shown in Fig. 5(a) retain their shape and therefore the resonance peak wavelength is easily measured. Fig. 5 (b) is presented to better illustrate the relationship between the measured shifts in the resonance peak wavelength position of the sensor and corresponding refractive indices of different organic solvents. It can be seen that the wavelength positions of the first resonance peak measured as a function of the refractive indices of organic solvents can be satisfactorily fitted by a second order polynomial function.

To assess the effect of bend diameter on sensitivity, two more sensors were fabricated with bend diameters of 19.3 and $18.7 \mathrm{~mm}$. Fig. 5(c) presents the measured wavelength shifts of the peaks for the three different bend diameters as a function of the RI of the surrounding liquid. The figure clearly shows that the sensitivity of the proposed refractometer depends on the diameter of the bend. The measured average sensitivities are $725.76 \mathrm{~nm} / \mathrm{RIU}$, $392.42 \mathrm{~nm} / \mathrm{RIU}$ and $268.18 \mathrm{~nm} / \mathrm{RIU}$ for $19.3,19$ and $18.7 \mathrm{~mm}$ bend diameters, respectively. From Fig. 5(c), one can also see that the average slope of the dependence of the measured peak wavelength versus RI increases with the bend diameter and thus the corresponding sensitivity is increased as well. It is worth noting that in general for bend loss sensors where WGM effects do not occur, it is expected that sensitivity will increase as the bend diameter decreases but in this case the reverse is true, in that bend diameters of $18.7 \mathrm{~mm}$ are less sensitive than $19.3 \mathrm{~mm}$. This is because of the nature of WGMs, which means that interference effects may increase around certain wavelengths as the bend diameter gets larger.
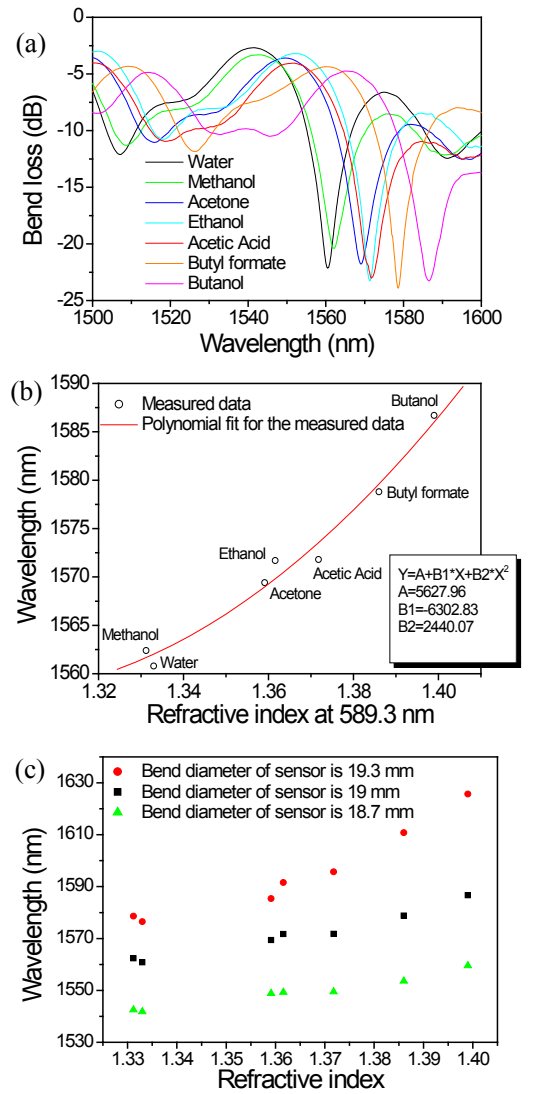

Fig. 5. (a) Transmission spectra of the fiber sensing device in a range of organic solvents; (b) $1^{\text {st }}$ resonance peak shifts as a function of corresponding RI (at 589.3nm); (c) the peak wavelength shifts versus RI.

In conclusion, an all-fiber refractometer specifically for low RI liquids (RI range of $1.33 \sim 1.40$ ) has been developed and investigated. The refractometer has a simple optical configuration and consists of a full-loop structure of coating stripped bare 1060XP singlemode fiber, employing the WGM effect. The developed bent bare fiber based refractometer shows a competitive sensitivity and does not require a complex fabrication process.

The support of the Irish Research Council for Science, Engineering and Technology (IRCSET) is gratefully acknowledged.

\section{References}

[1] I. Del Villar, I. R. Matias, and F. J. Arregui, Opt. Lett. 30, 2363 (2005).

[2] W. Liang, et al, Appl. Phys. Lett. 86, 151122 (2005).

[3] B. Gauvreau, et al, Opt. Express 15, 11413 (2007).

[4] T. Wei, et al, Opt. Express 16, 5764 (2008).

[5] P. Wang, et al, Applied Optics 48, 6044 (2009).

[6] J.Zhang, D.Grischowsky, JOSA B 20(9), 1894 (2003)

[7] S. H. Nam and S. Yin, IEEE Photon. Technol. Lett. 17, 2391 (2005).

[8] W. P. Huang, C. L. Xu, IEEE J. Quantum Electron. 29, 2639 (1993).

[9] P. Wang, et al, Proceeding of SPIE 7003, 7003-1Y (2008). 